Discrete Comput Geom 31:229-242 (2004)

DOI: $10.1007 / \mathrm{s} 00454-003-0807-6$

\title{
Linear Inequalities for Rank 3 Geometric Lattices
}

\author{
Kathryn L. Nyman \\ Department of Mathematics, Texas A\&M University, \\ College Station, TX 77843, USA \\ nyman@math.tamu.edu
}

\begin{abstract}
The flag Whitney numbers (also referred to as the flag $f$-numbers) of a geometric lattice count the number of chains of the lattice with elements having specified ranks. We give a collection of inequalities which imply all the linear inequalities satisfied by the flag Whitney numbers of rank 3 geometric lattices. We further describe the smallest closed convex set containing the flag Whitney numbers of rank 3 geometric lattices as well as the smallest closed convex set containing the flag Whitney numbers of those lattices corresponding to oriented matroids.
\end{abstract}

\section{Introduction}

Recall, for a geometric lattice $L$ of rank $n$, the Whitney numbers (of the second kind) are the numbers $\left\{\alpha_{\emptyset}(L), \alpha_{1}(L), \ldots, \alpha_{n}(L)\right\}$ where $\alpha_{k}(L)$ denotes the number of elements in $L$ of rank $k\left(\alpha_{\emptyset}=1\right)$. We define the flag Whitney numbers $\left\{\alpha_{S}\right\}, S \subseteq[n-1]$, to be the number of chains of $L$ which stop at ranks in $S$; namely, for $S=\left\{s_{1}, \ldots, s_{l}\right\}$,

$$
\alpha_{S}(L)=\left|\left\{y_{1}<y_{2}<\cdots<y_{l}: r\left(y_{i}\right)=s_{i}\right\}\right| .
$$

A second lattice invariant derived from the flag Whitney numbers is

$$
\beta_{S}(L)=\sum_{T \subseteq S}(-1)^{|S|-|T|} \alpha_{T}(L) .
$$

The invariants $\alpha_{S}$ and $\beta_{S}$ can be defined for any poset and are frequently referred to as the flag $f$-vector and flag $h$-vector of the poset. We use the term flag Whitney numbers exclusively for geometric lattices, while the flag $f$-vector is used for all other posets and line arrangements.

Determining which sequences of numbers arise as the flag $f$-vector of a poset is a difficult question. One step toward obtaining a characterization of these vectors is to 
determine the linear inequalities they must satisfy. Billera and Hetyei [2] answered this question for graded posets, finding there are Catalan many extreme rays in the closed cone generated by the flag $f$-vectors of all graded posets of a given rank. Although the corresponding minimal list of linear inequalities is not explicitly known, this list is necessarily finite. Stanley showed the flag $f$-vectors of graded Cohen-Macaulay posets satisfy $2^{n}$ linear inequalities. This result follows, for example, from [18, Theorem III: 4.4] and [3, Theorem 2]. A consequence of the $g$-theorem for simplicial convex polytopes (see [4] and [17]) is that all linear inequalities on the flag $f$-vectors of posets that are face lattices of simplicial polytopes (and by duality simple polytopes) are implied by $\lfloor d / 2\rfloor$ inequalities. Finally Kruskal [11] and Katona [10] showed that a finite number of inequalities are sufficient to describe all the linear inequalities satisfied by the flag $f$-vectors of face lattices of simplicial complexes. In this paper we give the minimal list of linear inequalities satisfied by the flag Whitney numbers of geometric lattices of rank 3 . Of interest is that while in the previous cases a finite number of inequalities were sufficient to imply all possible linear inequalities satisfied by the flag $f$-vectors, we find an infinite family of linear inequalities are necessary to imply all linear inequalities in the rank 3 geometric lattice case.

The following is a complete set of inequalities for the flag Whitney numbers $\left(\alpha_{1}, \alpha_{2}\right.$, $\alpha_{12}$ ) of geometric lattices of rank 3 . In the special case of line and pseudoline arrangements in the plane (discussed in Section 4), the additional inequality $\alpha_{12} \leq 3 \alpha_{2}-3$ completes the description.

Theorem 1. The closed convex set generated by all flag Whitney numbers of rank 3 geometric lattices is determined by the minimal set of inequalities

$$
\alpha_{12} \geq 2 \alpha_{2}, \quad \alpha_{2} \geq \alpha_{1}, \quad \alpha_{1} \geq 3,
$$

and, for $k \geq 3$,

$$
(k-1) \alpha_{12}-k \alpha_{1}-(2 k-3) \alpha_{2}+\left(\begin{array}{c}
k+1 \\
2
\end{array}\right) \geq 0 .
$$

\section{Extreme Lattices}

An arrangement of points in the plane along with all the lines determined by these points gives rise to a rank 3 geometric lattice. The points correspond to atoms of the lattice, and the lines to rank 2 elements. A rank 2 element covers an atom exactly when the corresponding point lies on the line (see Fig. 1). While not all rank 3 geometric lattices have a corresponding arrangement, for those that do the flag Whitney numbers $\alpha_{1}, \alpha_{2}$, and $\alpha_{12}$ give the number of points, lines, and point-line incidences in the arrangement. A natural duality exists between points and lines in the projective plane (see, e.g., [8]). Thus an arrangement of lines in the plane, every pair of which meets in a finite point, or at a point at infinity, also gives rise to a rank 3 geometric lattice. We use examples from both line arrangements and point arrangements in our discussions.

Define the $\alpha$-vector of a rank 3 geometric lattice to be the vector of flag Whitney numbers (excluding $\left.\alpha_{\emptyset}\right)\left(\alpha_{1}, \alpha_{2}, \alpha_{12}\right) \in \mathbb{R}^{3}$. We first note that a geometric lattice of 

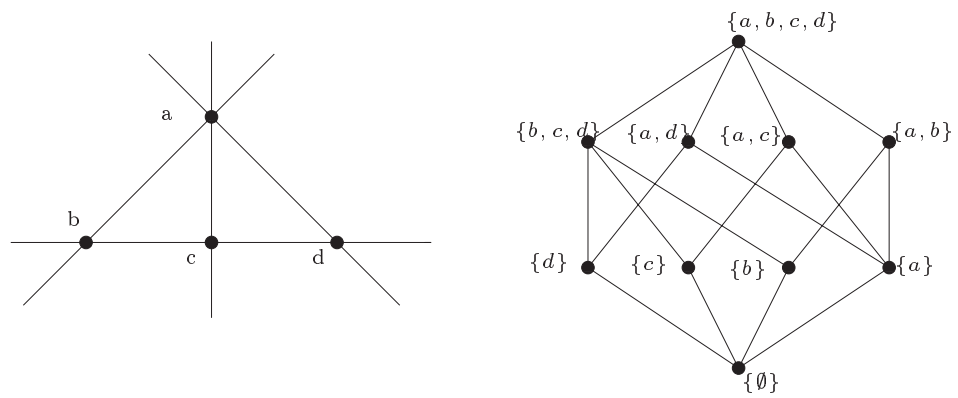

Fig. 1. The near pencil arrangement on four points in the plane with its corresponding geometric lattice.

rank 3 must have at least three atoms and at least three coatoms. Thus the "smallest" geometric lattice of rank 3 is the Boolean lattice $B_{3}$ on three points, which corresponds to the arrangement of three points in the plane, not all on a line. This is the only rank 3 geometric lattice having three atoms and so the $\alpha$-vector $(3,3,6)$ is the unique vector with $\alpha_{1}=3$.

Consider the geometric lattices which correspond to $n$ points in general position in the plane. These lattices are a truncation of the Boolean lattice $B_{n}$ at rank 3. Since the points are in general position each line contains exactly two points, and so we have $n$ points, $\left(\begin{array}{l}n \\ 2\end{array}\right)$ lines, and $2\left(\begin{array}{l}n \\ 2\end{array}\right)$ point-line incidences. We will find the $\alpha$-vectors $\left(n,\left(\begin{array}{l}n \\ 2\end{array}\right), 2\left(\begin{array}{l}n \\ 2\end{array}\right)\right)$ for $n \geq 3$ are extreme points in the smallest closed convex set containing the $\alpha$-vectors of all rank 3 geometric lattices.

Next, consider the geometric lattices corresponding to the near pencil arrangement on $n$ points in the plane (Fig. 1). This arrangement consists of $(n-1)$ two-point lines and one $(n-1)$-point line. The corresponding $\alpha$-vector is

$$
(n, n, 3(n-1))
$$

which can be written as $(3,3,6)+(n-3) \cdot(1,1,3)$. Thus each of these vectors lies on the ray in the direction of $(1,1,3)$ from $(3,3,6)$.

Although not representable by points and straight lines in the plane, $P G(2, q)$, the finite projective plane on $n=q^{2}+q+1$ points, for $q$ a prime power, gives rise to a rank 3 geometric lattice (see Fig. 2). $P G(2, q)$ has $q^{2}+q+1$ lines, each of which

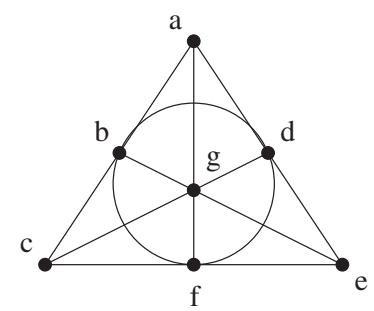

Fig. 2. The projective plane on $n=7$ points. 


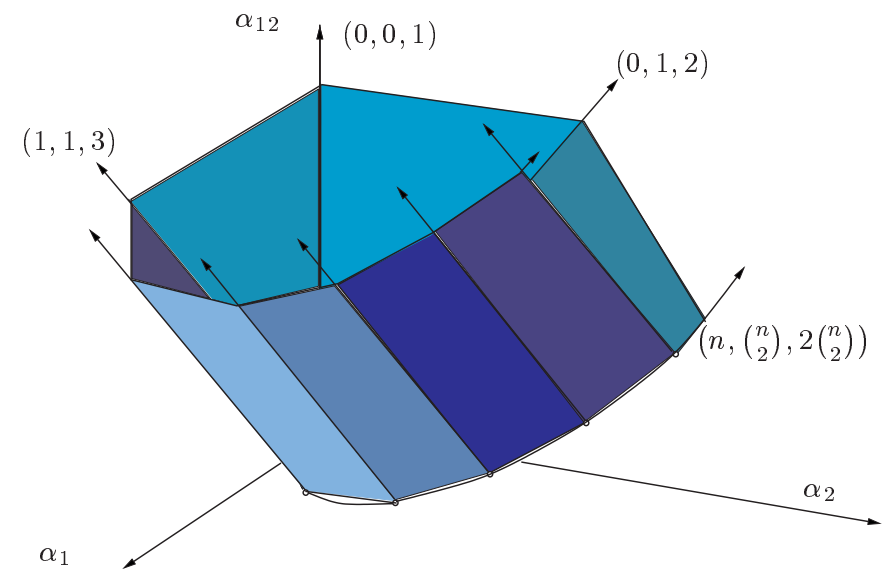

Fig. 3. The closed convex set containing the flag Whitney numbers of geometric lattices of rank 3 .

contains $q+1$ points, so the $\alpha$-vector of the associated geometric lattice is

$$
\left(q^{2}+q+1, q^{2}+q+1,(q+1)\left(q^{2}+q+1\right)\right) .
$$

Dividing by the highest power of $q$ appearing, we find these $\alpha$-vectors approach the direction $(0,0,1)$ from the point $(3,3,6)$ as $n$ (and consequently $q$ ) approaches $\infty$. Similarly, we find that the $\alpha$-vectors corresponding to $n$ points in general position have a limiting direction ray of $(0,1,2)$ from $(3,3,6)$ as $n \rightarrow \infty$.

A set of points and a set of direction rays determine a closed convex set consisting of the convex hull of the points plus the cone generated by the direction rays. It is straightforward to verify that the inequalities of Theorem 1 describe the closed convex set generated by the arrangements of the near pencils, the general position arrangements and the limits of the general position arrangements and finite projective planes. This closed convex set has extreme points $\left(n,\left(\begin{array}{l}n \\ 2\end{array}\right), 2\left(\begin{array}{l}n \\ 2\end{array}\right)\right)$, for $n \geq 3$ and extreme rays $(0,0,1)$, $(0,1,2)$, and $(1,1,3)$. It is illustrated (not to scale) in Fig. 3 in which the $\alpha_{1}, \alpha_{2}$, and $\alpha_{12}$ axes meet at the point $(3,3,6)$.

The examples in this section show the smallest closed convex set generated by the $\alpha$-vectors of rank 3 geometric lattices must contain the set of Theorem 1. To prove Theorem 1 we need only verify the inequalities hold.

\section{Inequalities on the Flag Whitney Numbers}

The following inequalities are well known:

$$
\alpha_{12} \geq 2 \alpha_{2}, \quad \alpha_{2} \geq \alpha_{1}, \quad \alpha_{1} \geq 3
$$

The first inequality follows from the fact that every line contains at least two points. $\alpha_{1} \geq 3$ is a consequence of three atoms being necessary to span a rank 3 geometric lattice, and $\alpha_{1} \leq \alpha_{2}$ follows from several classic results (see, e.g., [1]). 
To prove the remaining family of inequalities we utilize a combinatorial interpretation of the $\beta_{S}$ which involves shelling the balanced order complex of a geometric lattice. A description of the balanced order complex can be found in [18] and a discussion of this interpretation of $\beta_{S}$ can be found in [16, Proposition 3.5]. In rank 3 the balanced order complex of a geometric lattice is equivalent to the lattice without $\{\hat{0}, \hat{1}\}$ where the rank 1 elements (called points) are colored with color 1, and the rank 2 elements (lines) have color 2. A shelling of this resulting bipartite graph is an ordering of the edges $\left\{e_{1}, \ldots, e_{k}\right\}$ so that for any $i$, the partial graph consisting of $\left\{e_{1}, \ldots, e_{i}\right\}$ and all incident vertices is connected. As each edge is added to the ordering (shelled) it makes a contribution to $\beta_{j}$ where $j \in\{\emptyset, 1,2,12\}$ is the color of the minimal new face added (which is $\emptyset$ for the first edge added, and is the color 1 or 2 of the newest vertex added to the partial graph, or 12 if an edge is added between two vertices already present in the partial graph).

By the subgraph on atoms $\{1, \ldots, k\}$ we mean the subgraph consisting of the atoms $1, \ldots, k$, the lines $\{i \vee j: i, j \in\{1, \ldots, k\}\}$, and all edges between these points and lines. By adding an atom $k+1$ to the subgraph on $\{1, \ldots, k\}$ we mean extending the shelling from the subgraph on $\{1, \ldots, k\}$ to the subgraph on $\{1, \ldots, k+1\}$. We shell the bipartite graph as follows.

Shelling Method (*). For $L$ a rank $r$ geometric lattice with $n$ atoms, label the atoms $1, \ldots, n$ such that the atoms $\{1,2,3\}$ form a basis for the underlying matroid of $L$ (so $1 \vee 2 \vee 3=\hat{1}$ ), and such that if atom 1 is covered by $m$ lines, $2 \leq m \leq n-1$, these lines are given by $\{1 \vee i: i \in\{2, \ldots, m+1\}\}$. For each line $l$ assign a basis consisting of the two smallest labeled atoms whose join is $l$. We first shell the subgraph on the atoms $\{1,2,3\}$ as illustrated in Fig. 4. Shelling the subgraph on $\{1,2,3\}$ has a net contribution to $\left(\beta_{1}, \beta_{2}, \beta_{12}\right)$ of $(2,2,1)$.

We continue shelling the graph in stages; at each stage we add the next atom to the subgraph. The atoms $4, \ldots, m+1$ span lines with atom 1 . Suppose we have shelled the subgraph on atoms $\{1, \ldots, i-1\}$, where $i \in\{4, \ldots, m+1\}$. Let $j_{i}$ be the number of
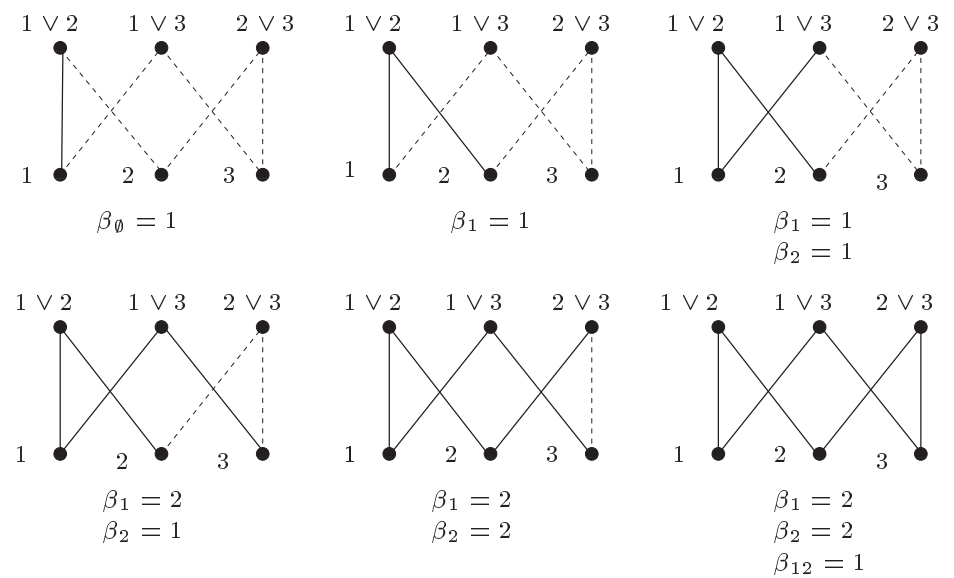

Fig. 4. Shelling the subgraph on atoms $\{1,2,3\}$. 


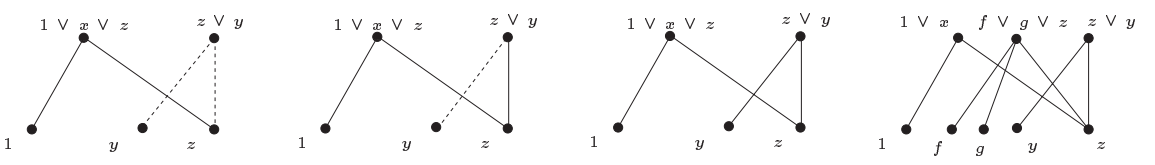

Fig. 5. Adding $z \in\{m+2, \ldots, n\}$ to the subgraph on $\{1, \ldots, z-1\}$.

lines spanned by the atoms $i$ and $h$, with $1<h<i$, henceforth called new $i$-lines, and let $l_{i}$ be the number of lines containing $i$, spanned by atoms $f$, $g$, with $1<f<g<i$ (old $i$-lines).

Shelling the edge from 1 to $1 \vee i$ contributes one to $\beta_{2}$ and shelling the edge from $1 \vee i$ to $i$ contributes one to $\beta_{1}$. Now for each new $i$-line $i \vee h$ we have a contribution to $\beta_{2}$ (from the edge $i$ to $i \vee h$ ) and a contribution to $\beta_{12}$ (from the edge $h$ to $i \vee h$ ). Each old $i$-line $f \vee g$ contributes one to $\beta_{12}$ when the edge from $i$ to $f \vee g$ is added. Note any such line $f \vee g$ along with edges between $f \vee g$ and $f$ and $g$ are already in the shelled subgraph on $\{1, \ldots, i-1\}$. Thus the net contribution to $\left(\beta_{1}, \beta_{2}, \beta_{12}\right)$ of adding $i \in\{4, \ldots, m+1\}$ to the subgraph is $\left(1,1+j_{i}, j_{i}+l_{i}\right)$.

We continue in a similar manner with atoms $\{m+2, \ldots, n\}$ as follows. Suppose we have shelled the subgraph on atoms $\{1, \ldots, z-1\}$ with $z \in\{m+2, \ldots n\}$. Each such $z$ lies on a line $1 \vee x$ for $x \in\{2, \ldots, m+1\}$. Adding the edge from $1 \vee x$ to $z$ makes a contribution to $\beta_{1}$. For each (new) $z$-line with basis $\{z, y\}, 1<y<z$, adding the edges from $z$ to $z \vee y$ and from $y$ to $z \vee y$ makes a contribution to $\beta_{2}$ and $\beta_{12}$. Finally adding edges between $z$ and any (old) lines covering $z$, spanned by atoms $f, g(1<f<g<z)$, makes a contribution to $\beta_{12}$ (see Fig. 5).

Say $z$ makes $j_{z}$ new lines and lies on $l_{z}$ old lines. Then the contribution to $\left(\beta_{1}, \beta_{2}, \beta_{12}\right)$ of adding $z \in\{m+2, \ldots, n\}$ to the subgraph is $\left(1, j_{z}, j_{z}+l_{z}\right)$. Since $m \geq 2$ and the line $1 \vee x$ covering $z$ contains exactly one of $\{2, \ldots, m+1\}$, either $j_{z}$ or $l_{z}$ must be at least 1 .

Proposition 2. For $L$ a geometric lattice of rank 3, and $k \in \mathbb{Z}_{+}$, the flag Whitney numbers $\alpha_{1}(L), \alpha_{2}(L), \alpha_{12}(L)$ satisfy

$$
(k-1) \alpha_{12}-k \alpha_{1}-(2 k-3) \alpha_{2}+\left(\begin{array}{c}
k+1 \\
2
\end{array}\right) \geq 0 .
$$

Each inequality in this family (for $k \geq 3$ ) determines a hyperplane containing the direction $(1,1,3)$ and two consecutive general position points $\left(k,\left(\begin{array}{l}k \\ 2\end{array}\right), 2\left(\begin{array}{l}k \\ 2\end{array}\right)\right)$ and $(k+1$, $\left.\left(\begin{array}{c}k+1 \\ 2\end{array}\right), 2\left(\begin{array}{c}k+1 \\ 2\end{array}\right)\right)$.

Proof. First notice that when $k=1$, (4) is implied by $\alpha_{1} \leq \alpha_{2}$. So we may assume $k \geq 2$. To take advantage of the properties of $\beta_{S}$, we change (4) into an inequality in $\beta_{S}$. Thus the inequality we wish to prove becomes

$$
(k-1) \beta_{12}-\beta_{1}-(k-2) \beta_{2} \geq-\frac{1}{2}\left(k^{2}-3 k+4\right) .
$$

Shell the bipartite graph of $L$ as indicated in shelling method $(*)$. The total contribution to $\left(\beta_{1}, \beta_{2}, \beta_{12}\right)$ from shelling the subgraph on $\{1,2,3\}$ is $(2,2,1)$, and so the contribution to the left of $(5)$ is $(-k+1)$. 
Adding each atom $z \in\{m+2, \ldots, n\}$ to the subgraph contributes

$$
(k-1)\left(j_{z}+l_{z}\right)-1-(k-2) j_{z}=j_{z}+l_{z}(k-1)-1 \geq 0
$$

to the left of (5), where $j_{z}$ is the number of new $z$-lines and $l_{z}$ is the number of old $z$-lines. The above expression is non-negative since either $j_{z}$ or $l_{z} \geq 1$ and we assume $k \geq 2$.

We have yet to account for the contribution to the left of (5) from adding the atoms $4, \ldots, m+1$ to the subgraph. Each atom $i \in\{4, \ldots, m+1\}$ contributes

$$
(k-1)\left(j_{i}+l_{i}\right)-1-(k-2)\left(j_{i}+1\right)=\left(l_{i}-1\right)(k-1)+j_{i}
$$

to the left of (5). This contribution is non-negative unless $l_{i}=0$ and $j_{i}<(k-1)$. Let $T \subseteq\{4, \ldots, m+1\}$ be the subset of atoms having $l_{i}=0$. All other atoms in $\{4, \ldots, m+1\}$ make a non-negative contribution to the left of (5). So the total contribution of the atoms $4, \ldots, m+1$ to the left of (5) is greater than or equal to the contribution from the atoms in $T$. Suppose $|T|=t$. Each atom of $T$ lies on no lines spanned by previous atoms. Furthermore, each $y \in T$ must make a new line with each atom $2, \ldots, y-1$ since any line covering $y$ containing two of $\{2, \ldots, y-1\}$ would have been shelled prior to shelling atom $y$ (and hence would be an old $y$-line). Thus $j_{y}=y-2$, and so

$$
\sum_{y \in T} j_{y} \geq(2+3+\cdots+(t+1))
$$

with the minimum occurring if $T=\{4,5, \ldots, 4+t-1\}$. Hence the total contribution of adding (to the subgraph) the atoms $4, \ldots, m+1$ to the left of (5) is at least

$$
\begin{aligned}
t(-k+1)+(2+3 \cdots+(t+1)) & =-t k+t+\left(\begin{array}{c}
t+2 \\
2
\end{array}\right)-1 \\
& =\frac{1}{2} t^{2}+\frac{5}{2} t-t k,
\end{aligned}
$$

where $0 \leq t \leq(n-3)$.

We find (6) is minimized when $t=k-\frac{5}{2}$. Since $t$ and $k$ are positive integers we check $f(t)=\frac{1}{2} t^{2}+\frac{5}{2} t-t k$ at $t=k-3$ and $t=k-2$ and find $f(k-3)=f(k-2)=$ $-\frac{1}{2} k^{2}+\frac{5}{2} k-3$. Thus the contribution of adding atoms $4, \ldots, m+1$ to the left of (5) is at least $-\frac{1}{2} k^{2}+\frac{5}{2} k-3$. Combining this with the contribution of $\{1,2,3\}$ and recalling the contribution of the atoms $m+2, \ldots, n$ is non-negative, we have that the total contribution to the left of (5) is at least

$$
-\frac{1}{2} k^{2}+\frac{5}{2} k-3-k+1=-\frac{1}{2} k^{2}+\frac{3}{2} k-2,
$$

which is the right of (5).

Proof of Theorem 1. Proposition 2 along with the inequalities $\alpha_{12} \geq 2 \alpha_{2}, \alpha_{2} \geq \alpha_{1}$, and $\alpha_{1} \geq 3$, which hold for all $\alpha$-vectors of geometric lattices of rank 3 , imply the closed convex set generated by the inequalities of Theorem 1 contains the closed convex set generated by the $\alpha$-vectors of all rank 3 geometric lattices. Furthermore, the geometric lattices corresponding to $n$ points in general position along with the extreme rays $(1,1,3)$, 
$(0,0,1)$, and $(0,1,2)$ corresponding to the near pencil arrangements, and the limits of finite projective planes and general position arrangements as $n \rightarrow \infty$, indicate the closed convex set generated by the $\alpha$-vectors for all rank 3 geometric lattices contains the convex set of Theorem 1.

Notice since the $\alpha$-vectors $\left(n,\left(\begin{array}{l}n \\ 2\end{array}\right), 2\left(\begin{array}{l}n \\ 2\end{array}\right)\right)$ for $n>3$ satisfy as equalities $\alpha_{12} \geq 2 \alpha_{2}$ and (4) for $k=n-1$ and $k=n$, the $\alpha$-vectors corresponding to the arrangements of $n$ points in general position are extreme points of the closed convex set of Theorem 1 (the vector $(3,3,6)$ satisfies $\alpha_{12}=2 \alpha_{2}, \alpha_{1}=3$ and (4) for $k=3$ ).

Remark 3. The set of flag Whitney numbers of rank 3 geometric lattices fails to be convex. The vectors $(7,7,21)$ and $(7,7,18)$ correspond to the projective plane on seven points and the near pencil arrangement on seven points, respectively, but it can be shown by a case analysis that there does not exist a geometric lattice with $\alpha$-vector $(7,7,20)$.

The next family of inequalities states that for geometric lattices with at least $k$ atoms, the flag Whitney numbers lie above an additional hyperplane which passes through the vertex $(3,3,6)$, the general position point $\left(k,\left(\begin{array}{l}k \\ 2\end{array}\right), 2\left(\begin{array}{l}k \\ 2\end{array}\right)\right)$, and includes the direction ray $(1,1,3)$. The hyperplanes corresponding to this family of inequalities are illustrated in Fig. 6.

Proposition 4. For L a geometric lattice of rank 3 with $n \geq k \geq 3$ atoms,

$$
\frac{1}{2} k \alpha_{12}-(k-1) \alpha_{2}-\left(\frac{1}{2} k+1\right) \alpha_{1}+\frac{3}{2} k \geq 0 .
$$

The inequalities of Proposition 4 cannot be strengthened since the hyperplane $\frac{1}{2} k \alpha_{12}-$ $(k-1) \alpha_{2}-\left(\frac{1}{2} k+1\right) \alpha_{1}+\frac{3}{2} k=0$ contains the points $(k, k, 3(k-1))$, and $\left(k,\left(\begin{array}{l}k \\ 2\end{array}\right), 2\left(\begin{array}{l}k \\ 2\end{array}\right)\right)$, along with the direction $(1,1,3)$. As in the proof of Proposition 2, the proof of Proposition 4 uses the shelling method (*) for a general rank 3 geometric lattice. A proof can be found in [14].

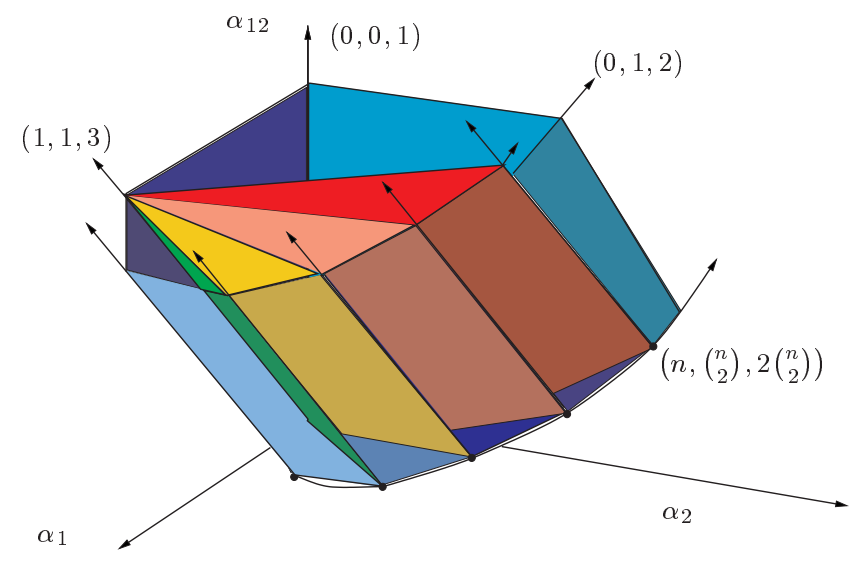

Fig. 6. The family of additional hyperplanes of Proposition 4. 


\section{Inequalities for Pseudoline Arrangements}

A pseudoline (see [5]) is a simple closed curve in the projective plane whose complement is connected. A pseudoline arrangement means a collection of pseudolines satisfying the following two properties. Each pair of pseudolines intersects in a unique point and they cross each other at this point, and the intersection of all of the pseudolines is empty. An arrangement of pseudolines decomposes the projective plane into a two-dimensional cell complex of vertices, edges, and 2-cells.

The rank 3 version of Folkman and Lawrence's Topological Representation Theorem [6] establishes a 1-1 correspondence between arrangements of pseudolines and simple rank 3 oriented matroids. Thus it is of interest to study the geometric lattices associated to pseudoline arrangements. Given a pseudoline arrangement we can form a geometric lattice whose atoms correspond to the pseudolines. Every pair of pseudolines meets in a unique point and this point corresponds to the unique rank 2 element of the lattice covering those atoms.

Recall the flag $f$-vector of a $d$-dimensional arrangement $\mathcal{A}$ is given by

$$
f_{S}(\mathcal{A})=\left|\left\{x_{1} \subset \cdots \subset x_{l}: \operatorname{dim} x_{j}=i_{j}\right\}\right|
$$

for $S \subseteq[d-1]$. Call an arrangement of pseudolines simplicial if all the 2-cells generated by the arrangement are triangles; specifically if $f_{02}=3 f_{2}$. An example of a simplicial arrangement is the near pencil arrangement of $n$ lines and $n$ points in the plane. The flag Whitney numbers of a geometric lattice give much information regarding how an arrangement of $n$ pseudolines breaks up the plane into regions. Specifically the following transformation will be useful [19]:

$$
n=\alpha_{1}, \quad f_{0}=\alpha_{2}, \quad f_{1}=\alpha_{12}, \quad f_{2}=\alpha_{12}-\alpha_{2}+1 .
$$

Lemma 5. The flag Whitney numbers of rank 3 geometric lattices associated to pseudoline arrangements satisfy

$$
\alpha_{12} \leq 3 \alpha_{2}-3
$$

Proof. Consider the vertices, edges, and 2-cells formed by the pseudoline arrangement. Each 2-cell has at least three sides and so $f_{02} \geq 3 f_{2}$ with equality holding in the case when the arrangement is simplicial. Now $f_{02}=f_{01}=2 f_{1}$ since a point on $k$ edges is in $k$ 2-faces, and each edge of the arrangement has two endpoints. Thus $2 f_{1} \geq 3 f_{2}$. By the transformation of (7) we have $3 \alpha_{2} \geq \alpha_{12}+3$ as desired.

Inequality (8) implies for a geometric lattice associated to a pseudoline arrangement

$$
\frac{\alpha_{12}}{\alpha_{2}} \leq 3-\frac{3}{\alpha_{2}} .
$$

Thus the average number of lines on a point is less than three. This excludes, for example, the finite projective planes on $q^{2}+q+1$ points with $q$ a prime power from being pseudoline arrangements since each point lies on $(q+1)$ lines. Hence $\alpha_{12} / \alpha_{2}=q+1 \geq 3$. 


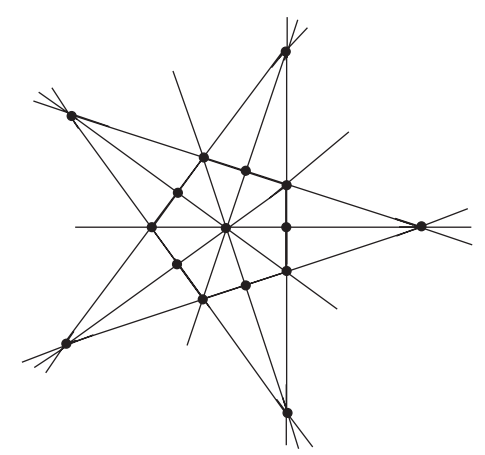

Fig. 7. The arrangement $R(10)$.

Remark 6. The pseudoline arrangements whose $\alpha$-vectors $\left(\alpha_{1}, \alpha_{2}, \alpha_{12}\right)$ lie on the hyperplane $\mathcal{H}$ through $(3,3,6)$ and containing the directions $(1,1,3)$ and $(0,1,3)$ are simplicial. This can be seen since $\mathcal{H}$ is exactly the hyperplane $\alpha_{12}=3 \alpha_{2}-3$ which we noted is satisfied by arrangements of pseudolines exactly when the arrangement is simplicial.

Example 7. The following infinite families of arrangements are simplicial and lie on the hyperplane $\mathcal{H}$. More details of these and other simplicial arrangements can be found in [8]:

- The near pencil arrangements on $n$ lines (equivalently on $n$ points) all lie on the ray $(3,3,6)+k(1,1,3) ; k \geq 0$.

- The arrangements formed by taking the $k$ lines determined by the edges of a regular $k$-gon along with its $k$ axes of symmetry are referred to as $R(2 k)$ for $k \geq 3$. $R(10)$ is illustrated in Fig. 7. The $\alpha$-vectors for these arrangements (see [7]) are

$$
\left(2 k,\left(\begin{array}{c}
k+1 \\
2
\end{array}\right)+1, \frac{3 k(k+1)}{2}\right)
$$

which approach the ray $(0,1,3)$ as $k \rightarrow \infty$.

- The arrangements $R(4 k+1)$ for $k \geq 2$ consist of the $4 k$ lines of $R(4 k)$ (see the above arrangement) plus the line at $\infty$. The $\alpha$-vectors of these arrangements (see [7]) are

$$
\left(4 k+1,2 k^{2}+2 k+1,6 k(k+1)\right)
$$

which also approach $(0,1,3)$ as $k \rightarrow \infty$.

We again note that in an arrangement of pseudolines we are taking the lines as atoms and the intersection points as rank 2 elements of the geometric lattice. The simplicial nature of an arrangement on $n$ points is not necessarily obvious from the picture.

Proposition 8. The closed convex set generated by all $\alpha$-vectors of rank 3 orientable geometric lattices (pseudoline arrangements) is determined by the set of inequalities of 


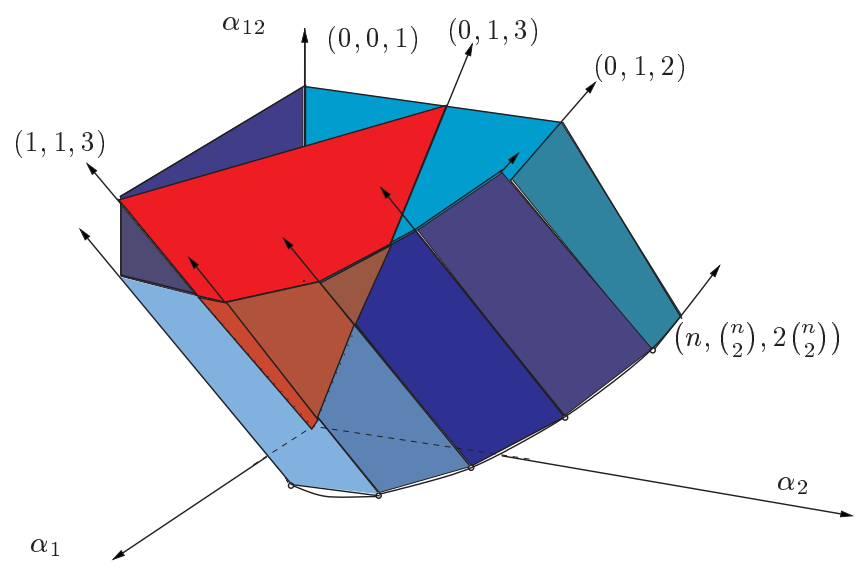

Fig. 8. The additional inequality bounding the $\alpha_{S}$ of geometric lattices corresponding to oriented matroids.

Theorem 1 together with the inequality

$$
\alpha_{12} \leq 3 \alpha_{2}-3
$$

This convex set is depicted in Fig. 8.

Proof. By Lemma 5 we know the $\alpha$-vectors of all pseudoline arrangements lie in the subspace $\alpha_{12} \leq 3 \alpha_{2}-3$. To show $\alpha_{12} \leq 3 \alpha_{2}-3$ gives the tightest possible inequality we consider the arrangements in Example 7 and observe these pseudoline arrangements, which lie on the hyperplane $\alpha_{12}=3 \alpha_{2}-3(\mathcal{H})$, approach the rays $(0,1,3)$ and $(1,1,3)$ as $k, n \rightarrow \infty$.

Remark 9. Although all rank 3 geometric lattices whose $\alpha$-vectors violate the inequality $\alpha_{12} \leq 3 \alpha_{2}-3$ correspond to non-orientable matroids, satisfying (8) is not a sufficient condition for orientability. For example, the MacLane matroid $M L_{8}$ (Fig. 9), obtained

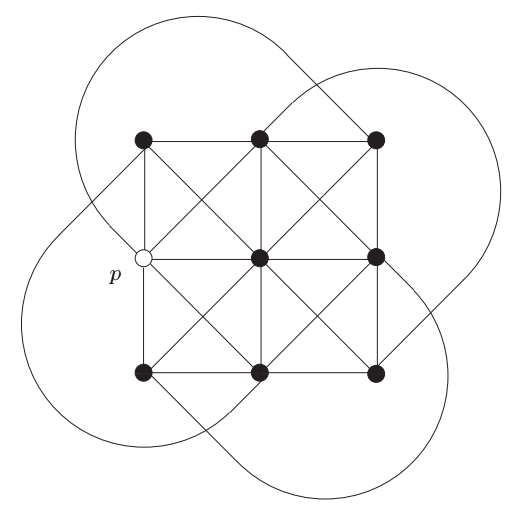

Fig. 9. A minimal non-orientable matroid $M L_{8}$. 
from the affine plane $A G(3,3)$ by removing one point $p$, has $\alpha_{1}=8, \alpha_{2}=12$, and $\alpha_{12}=32$ and so satisfies $\alpha_{12} \leq 3 \alpha_{2}-3$, but $M L_{8}$ is not orientable [12].

\section{Connections to a Result of Martinov}

In [13] Martinov gives a characterization of the number of 2-cells which can be induced by a pseudoline arrangement of a given number of lines [13, Theorem 2]. Specifically, there exists an arrangement with $n$ lines and $f_{2}$ 2-cells if and only if there exists an integer $k$, with $0 \leq k \leq n-2$, such that

$$
(n-k)(k+1)+\left(\begin{array}{l}
k \\
2
\end{array}\right)-\min \left\{n-k,\left(\begin{array}{l}
k \\
2
\end{array}\right)\right\} \leq f_{2} \leq(n-k)(k+1)+\left(\begin{array}{l}
k \\
2
\end{array}\right) .
$$

Translating Martinov's inequalities into inequalities on the flag Whitney numbers (via (7)) gives the following collection of lower and upper bounds on the quantity $\left(\alpha_{12}-\alpha_{2}+1\right)$ :

$$
\begin{aligned}
& \alpha_{12}-\alpha_{2}+1 \geq \alpha_{1} k-\left(\begin{array}{c}
k+1 \\
2
\end{array}\right), \\
& \alpha_{12}-\alpha_{2}+1 \geq \alpha_{1}(k+1)-k(k+1), \\
& \alpha_{12}-\alpha_{2}+1 \leq \alpha_{1}(k+1)-\frac{1}{2}\left(k^{2}+3 k\right),
\end{aligned}
$$

where $1 \leq k \leq n-2$. (We omit $k=0$ since this gives $f_{2}=n$ which corresponds to the the arrangement of $n$ lines with a common point. This arrangement does not give a rank 3 lattice.) These inequalities complement those which determine the closed convex set containing the $\alpha$-vectors of rank 3 orientable geometric lattices.

Figure 10 illustrates Martinov's inequalities for $n=6$ along with a cross section (at $\alpha_{1}=6$ ) of the closed convex set of Proposition 8 . The polyhedral region given by the inequalities of (8) and Theorem 1 for $\alpha_{1}=6$ is shaded while Martinov's constraints on $\alpha_{12}-\alpha_{2}+1$ consist of the bold lines and the striped region between the upper two bold lines. The $\alpha$-vectors corresponding to arrangements on six pseudolines are indicated with dots. From Martinov's work we see that for $n=6$ there exists an orientable geometric lattice such that $\alpha_{12}-\alpha_{2}=x$ for any $11 \leq x \leq 15$ or $x=9$. From this work we have additional constraints on $\alpha_{12}$ and $\alpha_{2}$, namely, that the pairs $\left(\alpha_{2}, \alpha_{12}\right)$ must also lie in the shaded region of Fig. 10.

Remark 10. It should be noted that satisfying Martinov's inequalities on $\left(\alpha_{12}-\alpha_{2}+1\right)$ does not guarantee that a corresponding geometric lattice exists. For example the vector $(6,12,26)$ satisfies Martinov's inequalities for $k=3$ and lies in the convex set of Proposition 8, however there does not exist a pseudoline arrangement with 6 lines and 12 points [8].

Remark 11. The set of flag Whitney numbers of rank 3 orientable geometric lattices also fails to be convex since the vectors $(6,6,15)$ and $(6,8,19)$ arise from line arrange- 


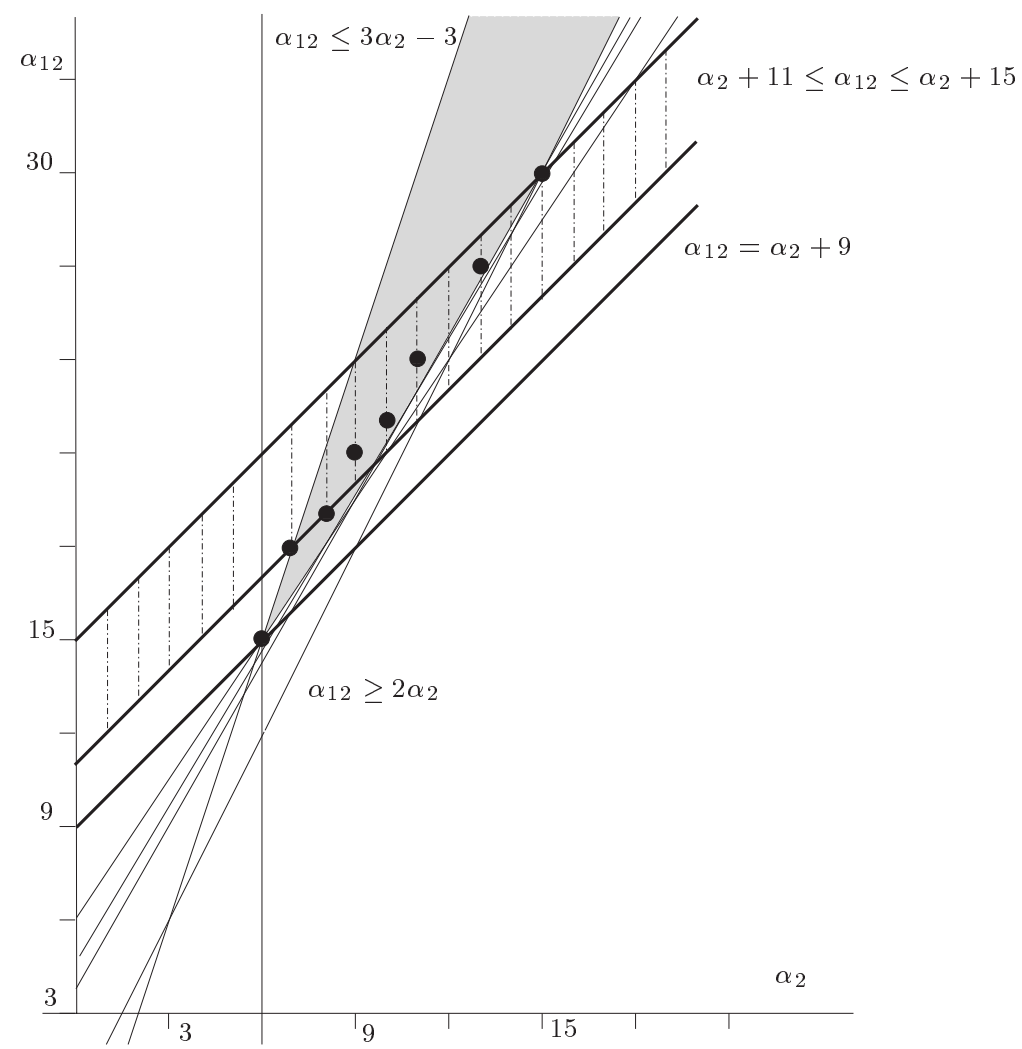

Fig. 10. Martinov's constraints for $n=6$ along with a cross section of the closed convex set of Proposition 8 at $\alpha_{1}=6$.

ments, while the vector $(6,7,17)$ fails Martinov's inequalities and thus can have no corresponding arrangement.

\section{Higher Ranks}

As we move from rank 3 to higher ranks, finding the convex hull containing the $\alpha$ vectors of geometric lattices is more complicated. However, some results from rank 3 hold for higher rank lattices. For example, the extreme ray obtained as the limit of the finite projective planes generalizes to higher dimensions. Let $P G(r-1, q)$ denote the $(r-1)$-dimensional projective space over $\mathbb{F}_{q}$. The $\alpha$-vector of the corresponding lattice can be calculated using formulas describing the subspaces of $P G(r-1, q)$ (see [9]). For example, $\alpha_{1,2, \ldots, r-1}=\left(q^{r}-1\right)\left(q^{r-1}-1\right) \cdots\left(q^{2}-1\right)(q-1) /(q-1)^{r-1}$. One may see that the limit, as $q \rightarrow \infty$, of the $\alpha$-vectors of such lattices gives the extreme ray $(0,0, \ldots, 0,1)$.

In addition recent work [15] shows that lattices corresponding to $n$ points in general position (exactly $d$ points in each $(d-1)$-dimensional subspace) in $(r-1)$-space 
maximize $\alpha_{S}$ for $S \subseteq[r-1]$, given $\alpha_{1}=n$. Finally, in [14] we see that lattices corresponding to generalized near pencil arrangements on $n$ points $((n-r+2)$ points on a line and $(r-2)$ points in general position) minimize $\alpha_{S}$ for $S \subseteq[r-1]$ given $\alpha_{1}=n$.

\section{Acknowledgments}

This work was done as part of my dissertation under the direction of Prof. L.J. Billera. I thank him for his guidance and suggestions.

\section{References}

1. J.G. Basterfield and L.M. Kelly, A characterization of sets of $n$ points which determine $n$ hyperplanes, Math. Proc. Cambridge Philos. Soc. 64 (1968), 585-588.

2. L.J. Billera and G. Hetyei, Linear inequalities for flags in graded partially ordered sets, J. Combin. Theory Ser. A 89 (2000), 77-104.

3. L.J. Billera and G. Hetyei, Decompositions of partially ordered sets, Order 17 (2000), 141-166.

4. L.J. Billera and C. Lee, Sufficiency of McMullen's conditions for $f$-vectors of simplicial polytopes, Bull. Amer. Math. Soc. 2 (1980), 181-185.

5. A. Björner, M. Las Vergnas, B. Sturmfels, N. White, and G.M. Ziegler, Oriented Matroids, Encyclopedia of Mathematics, Vol. 46, Cambridge University Press, Cambridge, 1999.

6. J. Folkman and J. Lawrence, Oriented matroids, J. Combin. Theory Ser. B 25 (1978), 199-236.

7. B. Grünbaum, Arrangements of hyperplanes, Congressus Numer. 3 (1971), 41-106.

8. B. Grünbaum, Arrangements and Spreads, AMS Regional Conference Series in Mathematics 10. AMS, Providence, RI, 1972.

9. J.W.P. Hirschfeld, Projective Geometries over Finite Fields, Oxford Mathematical Monographs, Clarendon Press, Oxford, 1998.

10. G. Katona, A theorem for finite sets, in Theory of Graphs (P. Erdös and G. Katona, eds.), Academic Press, New York, 1968, pp. 187-207.

11. J. Kruskal, The number of simplices in a complex, in Mathematical Optimization Techniques (R. Bellman, ed.), University. of California Press, Berkeley/Los Angeles, CA, 1963, pp. 251-278.

12. S. MacLane, Some interpretations of abstract linear independence in terms of projective geometry, Amer. J. Math. 58 (1936), 236-241.

13. N. Martinov, Classification of arrangements by the number of their cells, Discrete Comput. Geom. 9 (1993), 39-46.

14. K.L. Nyman, Enumeration in Geometric Lattices and the Symmetric Group, Ph.D. thesis, Cornell University, Ithaca, NY, 2001.

15. K.L. Nyman and E. Swartz, Inequalities for the $h$ - and flag $h$-vectors of geometirc lattices, preprint (2003).

16. R.P. Stanley, Balanced Cohen Macaulay complexes, Trans. Amer. Math. Soc. 249 (1979), 139-157.

17. R.P. Stanley, The number of faces of a simplicial convex polytope, Adv. in Math. 35 (1980), 236-238.

18. R.P. Stanley, Combinatorics and Commutative Algebra 2nd edition, Progress in Mathematics, Vol. 41, Birkhäuser, Boston, MA, 1996.

19. T. Zaslavsky, Facing up to arrangements: face count formulas for partitions of space by hyperplanes, Mem. Amer. Math. Soc. 154(1) (1975), vii+102 pp.

Received October 9, 2002, and in revised form April 4, 2003. Online publication December 19, 2003. 